\title{
Derivação gastrojejunal com anel de contenção no tratamento de obesidade mórbida em paciente com situs inversus totalis
}

\author{
The gastric bypass banding for morbid obesity in a patient with situs inversus
}

Cássio Jerônimo Machado de Barros'; ; Abrão Rapoport, ECBC-SP1; Josias de Andrade Sobrinho, ECBC-SP';

Rogério Aparecido Dedivitis, TCBC-SP1; Eduardo Halfen Grill ${ }^{1}$

\section{INTRODUÇÃO}

Situs inversus totalis é uma entidade rara, com predispoSição genética, que pode ocasionar dificuldades no tratamento de enfermidades abdominais, especialmente em cirurgia laparoscópica, devido à anatomia de imagem em espelho.

Nesse relato a técnica de derivação gastrojejunal em $Y$ de Roux, com anel de contenção, por via laparoscópica,é indicada em paciente obeso mórbido com cirurgia abdominal prévia e Situs Inversus Totalis.

\section{RELATO DO CASO}

Paciente de 24 anos, gênero masculino, peso de $149 \mathrm{~kg}$ no momento da cirurgia, com 1,93 m.de altura, índice de massa corporal de $40 \mathrm{Kg} / \mathrm{m}^{2}$, apresentava hipertensão arterial sistêmica controlada com medicamento. Foi indicado tratamento cirúrgico para obesidade mórbida, segundo critérios referendados pela Federação Internacional para a Cirurgia da Obesidade1. Durante a avaliação préoperatória, o paciente informou ser portador de Situs Inversus Totalis. Ao exame físico, apresentava cicatriz cirúrgica paramediana direita decorrente de apendicectomia complicada realizada previamente. Realizada avaliação cardiologia e pneumológica, sendo liberado para o procedimento cirúrgico.

Foram realizados tomografia computadorizada de abdômen (Figura 1) e radiografia contrastada do trânsito intestinal (Figura 2).

Optou-se pela gastroplastia em Y de Roux, com anel de contenção, conforme descrito por Capella et al. ${ }^{2}$. Devido aos benefícios da cirurgia minimamente invasiva, optamos por iniciar o procedimento por via laparoscópica.

A equipe posicionou-se em posição inversa à de rotina para gastroplastia videolaparoscópica. O cirurgião ficou à esquerda do paciente, juntamente com o auxiliar que controla a óptica, enquanto o assistente e a instrumentadora posicionaram-se à direita.

Iniciou-se com uma punção, utilizando trocarte ENDOPATH ${ }^{\mathrm{TM}} \times C \mathrm{XL}^{\mathrm{R}}{ }^{*}$, em hipocôndrio direito, afastada da incisão paramediana direita.
Ao inventário da cavidade abdominal, foi confirmada a inversão do posicionamento dos órgãos intraabdominais, associada à aderência da cirurgia prévia, sendo realizada lise cuidadosa das mesmas, com bisturi Ultracision $^{\mathrm{R}}$. Foi criada a bolsa gástrica de volume estimado de $50 \mathrm{ml}$, com endogrampeador de $45 \mathrm{~mm}$, e fixado anel de contenção em torno da nova câmara gástrica. Seguiu-se a secção do intestino delgado a aproximadamente $50 \mathrm{~cm}$ do ângulo de Treitz, localizado a direita da coluna vertebral. A alça proximal ou bíleo-pancreática foi anastomosada látero-lateralmente a proximadamente 100 $\mathrm{cm}$ da alça distal ou alça alimentar. A seguir, foi feita a transposição da alça alimentar em posição retro-gástrica, transmesocólica e fixação da extremidade da alça alimentar em parede lateral da bolsa gástrica, reforçando linha de grampeamento e fixando anel de contenção, para evitar seu escorregamento. Foi realizada gastro-ênteroanastomose com endogrampeador linear cortante, com teste das suturas através da infusão de solução de azul de metileno pela sonda gástrica, até dilatação da área de anastomose, não ocorrendo extravasamento. A seguir, os orifícios criados no mesocólon e no mesentério foram fe-

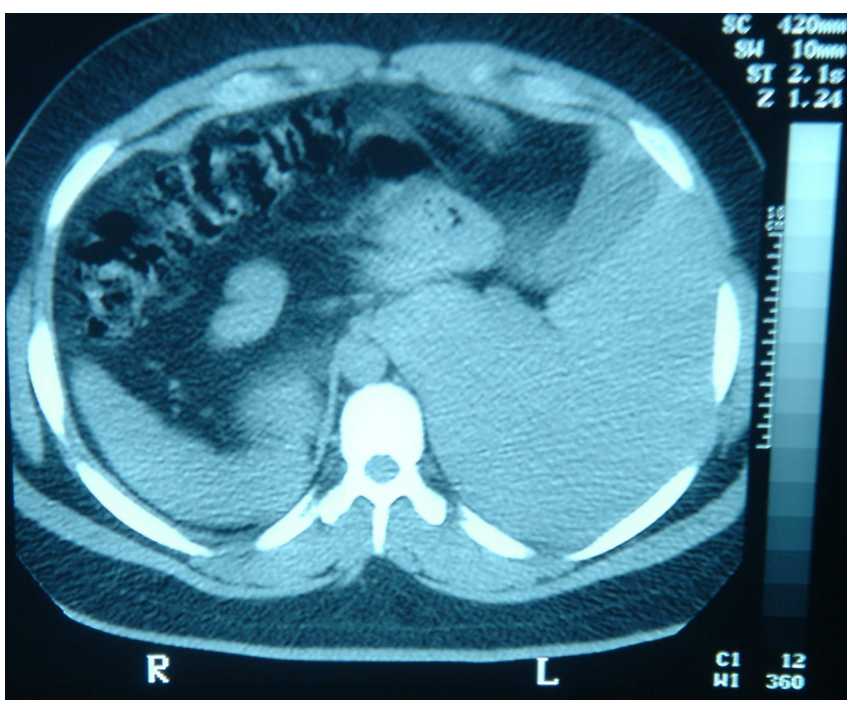

Figura 1 - Tomografia computadorizada de abdômen, mostrando Fígado e vesícula no lado direito e baço à esquerda no abdômen. 


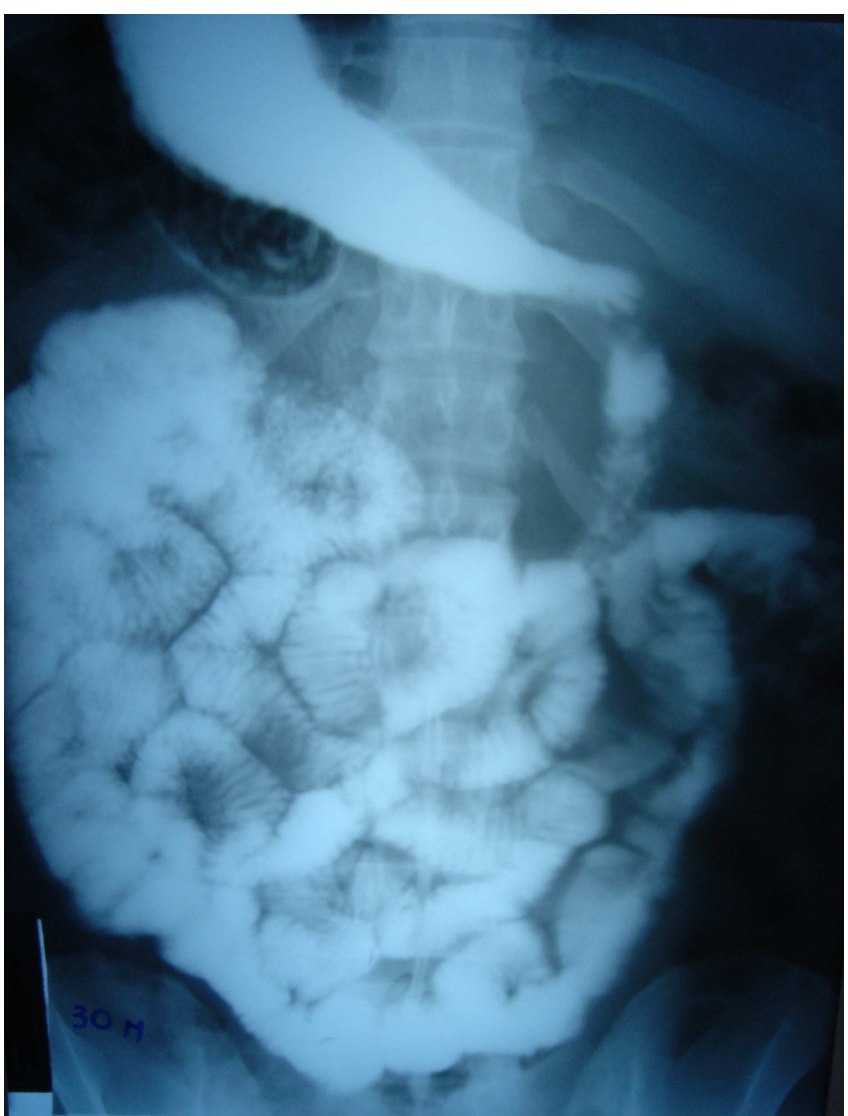

Figura 2 - Radiografia contrastada do trânsito intestinal, mostrando a grande curvatura gástrica voltada para a direita e o ângulo de Treitz à direita da coluna vertebral.

chados. O tempo cirúrgico foi de aproximadamente 400 minutos. Paciente apresentou boa evolução em seguimen- to ambulatorial, com perda ponderal de aproximadamente $30 \%$ em seis meses.

\section{DISCUSSÃO}

A cirurgia minimamente invasiva é uma opção oportuna no tratamento de enfermidades cada vez mais complexas, incluindo a obesidade mórbida ${ }^{3}$.

Foram encontrados dois relatos de tratamento cirúrgico de obesidade mórbida em paciente com situs inversus, ambos abordados por via laparoscópica. Em um deles, utilizou-se derivação gástrica laparoscópica sem uso de anel de contenção ${ }^{2}$, e no outro, empregou-se banda gástrica laparoscópica ${ }^{4}$. Não encontramos caso de gastroplastia em Y de Roux, com anel de contenção, conforme proposto por Capella et al. ${ }^{2}$ na técnica aberta ou na laparoscópica conforme Szegö et al. ${ }^{5}$, em paciente com Situs Inversus Totalis.

O tempo cirúrgico de 400 minutos é prolongado, porém, justificado pela situação peculiar, por ser uma situação anatômica rara, fora da rotina. Entretanto, os benefícios da cirurgia minimamente invasiva para o paciente foram evidentes, não necessitando de internação em unidade de terapia intensiva, não recebendo hemotransfusão e apresentando deambulação precoce, com alta hospitalar precoce.

Pacientes obesos mórbidos com Situs Inversus Totalis podem ser submetidos à gastroplastia em Y de Roux, com anel de contenção, por via laparoscópica. Cuidados especiais devem ser tomados no posicionamento das linhas de grampeamento e para não ocorrer inversão de alças, durante o processo de reconstrução do trânsito intestinal.

\section{A B S T R A C T}

The gastric bypass is a good option in the therapy of morbid obesity. Nevertheless, it must be considered the rare condition as occurred in a patient with previous abdominal surgery with Situs Inversus Totalis. A 24 year-old male patient with body mass index of $40 \mathrm{Kg} / \mathrm{m}^{2}$, multiple dietary failures, and arterial hypertension as co-morbidities, with a anterior paramedial right incision due to a previous appendicectomy (8 years ago). With a indication for bariatric surgery, was performed Roux-en-Y gastric bypass by laparoscopic procedure, with previous planning of Situs Inversus Totalis.

Key words: Situs Inversus. Gastric bypass. Bariatric surgery. Morbid obesity. Laparoscopy.

\section{REFERENCIAS}

1. International Federation for the Surgery of Obesity. Statement on patient selection for bariatric surgery. Obes Surg. 1997;7(1):41.

2. Capella RF, Capella JF, Mandae H. Vertical banded gastroplasty Gastric bypass: preliminary report. Obes Surg. 1991;1(4):389-95.

3. Wittgrove AC, Clark GW. Laparoscopic gastric bypass for morbid obesity in a patient with Situs Inversus. J Laparoendosc Adv Surg Tech A. 1998;8(1):53-5.

4. Ersoy E, Koksal H, Ege B. Laparoscopic gastric banding for morbid obesity in a patient with situs inversus totalis. Obes Surg. 2005;15(9):1344-6.

5. Szegö T, Mendes CJL, Bitran A. Derivação gastrojejunal por laparoscopia com e sem anel. In: Garrido Jr. AB. Cirurgia da obesidade. São Paulo: Atheneu; 2004. p.189-96.
Recebido em 05/06/2006

Aceito para publicação em 20/07/2006

Conflito de interesse: nenhum

Fonte de financiamento: nenhuma

\section{Como citar esse artigo:}

Barros CJM, Rapoport A, Andrade Sobrinho J, Dedivitis RA, Grill EH. Derivação gastrojejunal com anel de contenção no tratamento de obesidade mórbida em paciente com situs inversus totalis*. Rev Col Bras Cir. [periódico na Internet] 2010; 37(2). Disponível em URL: http:/ /www.scielo.br/rcbc

\section{Endereço para correspondência:}

Abrão Rapoport

E-mail: arapoport@terra.com.br 